\title{
Antibacterial Effects and Synthesized New Derivatives of 4-Hydroxy-Chromen-2-One
}

\author{
Aziz Behrami, Florent Dobroshi \\ Department of Food Technology, University of Mitrovica "Isa Boletini”, Mitrovica, Kosova
}

Email address:

a.behrami_chem@live.com (A. Behrami)

To cite this article:

Aziz Behrami, Florent Dobroshi. Antibacterial Effects and Synthesized New Derivatives of 4-Hydroxy-Chromen-2-One. American Journal of Heterocyclic Chemistry. Vol. 5, No. 1, 2019, pp. 7-10. doi: 10.11648/j.ajhc.20190501.13

Received: January 28, 2019; Accepted: March 21, 2019; Published: April 15, 2019

\begin{abstract}
In present paper, we report the organic syntheses of three compounds exactly 4-hydroxy-chromen-2-one and describe the results of antibacterial activity of purified compounds. Compounds 4-(4-Methoxy-phenylamino)-2-oxo-2Hchromene-3-sulfonyl chloride (a). N-\{3-[Diethoxy-(2-hydroxy-phenyl)-methyl]-2-oxo-2H-chromen-4-yl $\}$-acetamide (b), 4-(6Nitro-benzothiazol-2-ylamino)-2-oxo-2H-chromene-3-carbaldehyde (c), have been synthesized and characterized using melting points, IR spectra, ${ }^{1} \mathrm{H}-\mathrm{NMR}$ and ${ }^{13} \mathrm{C}$-NMR spectra. Our research goal is synthesis novel organic compounds which prove high level of antibacterial activity. The antibacterial activity of synthesized compounds and streptomycin and cefalexine at concentrations of $2 \mathrm{mg} / \mathrm{ml}, 3 \mathrm{mg} / \mathrm{ml}$ and $5 \mathrm{mg} / \mathrm{ml}$, have been evaluated against three strains of bacterial culture; Staphylococcus aureus, E. coli and Bacillus cereus. The compounds show bacteriostatic and bactericidal activity in high level and also synthetized compounds has been characterized using advanced instrumental methods of analysis.
\end{abstract}

Keywords: Coumarine Derivatives, Antibacterial Activity, IR, ${ }^{1} \mathrm{H}-\mathrm{NM},{ }^{13} \mathrm{C}-\mathrm{NMR}$, Streptomycine

\section{Introduction}

Starting from 4-hydroxy-chromen-2-one; derivatives (a, b, c) are synthesized. Coumarin derivatives are large group of heterocyclic with oxygen as heteroatom. Coumarin is a chemical compound (specifically, a benzo- $\alpha$-pyrone) found in many plants notably in high concentration in the tonka bean (Dipteryx odorata), vanilla grass (Anthoxanthum odoratum), woodruff (Galium odoratum), mullein (Verbascum spp), and sweet grass (Hierochloe odorata). Coumarine and their derivatives have shown varius biological activities. Their fame has come mainly from their antithrombic, antiinflammatory, vasodilatory, and antiviral activities. Other several coumarin derivatives have antimicrobial properties [12-15] with reflux and condensation we have synthesize some new coumarin derivatives and to investigate their antibacterial activity against Staphylococcus aureus, E. coli and Bacillus cereus. The antibacterial activity of synthesized compounds is compared with antibacterial activity of Cefalexine and Streptomycine [1-11].

\section{Materials and Methods}

\subsection{Experimental Chemistry}

4-(4-Methoxy-phenylamino)-2-oxo-2H-chromene-3sulfonyl chloride (a), N-\{3-[Diethoxy-(2-hydroxy-phenyl)methyl]-2-oxo-2H-chromen-4-yl $\}$-acetamide (b), 4-(6-Nitrobenzothiazol-2-ylamino)-2-oxo-2H-chromene-3carbaldehyde (c).

\subsection{Measurement}

The identification of Benzene - 1,3,5-triol derivatives (a, $\mathrm{b}, \mathrm{c})$, is made by using melting point, IR, $1 \mathrm{H} \mathrm{NMR},{ }^{13} \mathrm{C}$ NMR spectra and elemental analysis. Melting point was determinated on a Electrothermal apparatus (Fisher Scientific 2555) in a open capillary tube and are uncorrected. Infrared spectra were recorded in $\mathrm{cm}-1$ for $\mathrm{KBr}$ pellts on a FT-IR Shimadzu 8400S spectrophotometer with resolution $4 \mathrm{~cm}-1$. ${ }^{1} \mathrm{H}$ NMR spectra were recorded on a Bruker UNITY plus-500 'NMR 1' spectrometer using DMSO-d6 as the solvent and TMS as the internal references standard $(\sigma=0,00 \mathrm{ppm})$. Chemical shifts are expressed in $\delta$ ppm. Mass spectra were 
taken on a LKB 9000 mass spectrometer. Element analysze was performed on a Perikin-Elmer $240 \mathrm{BCHN}$ analyzer. The purity of the compounds (synthesized) was routinely checked by TLC using Merck Kieselgel-60 (F-254) and benzene, toluene, glacial acetic acid (80:10:10)as mobile phase. The spots were exposed in iodine vapour for visualization.

Preparation of 4-(4-Methoxy-phenylamino)-2-oxo-2Hchromene-3-sulfonyl chloride (a)
For this synthesis is used as substrat 4g 4- hydroxychromen-2-one in a $100 \mathrm{ml}$ flask mixed $2 \mathrm{~g}$ Anisidine, $8 \mathrm{ml}$ acetonitrile, $3 \mathrm{mlClSO}_{3} \mathrm{H}$ and $1 \mathrm{ml} \mathrm{Et}_{3} \mathrm{~N}$. The mixture was refluxed at $90^{\circ} \mathrm{C}$ for ca. $4 \mathrm{~h}$. The obtained crystals white are filtered and rinsed with methanol and dried at room temperature. Recrystalization form absolute methanol gave a white product of $70 \%$ yield, melting point $310^{\circ} \mathrm{C}$.

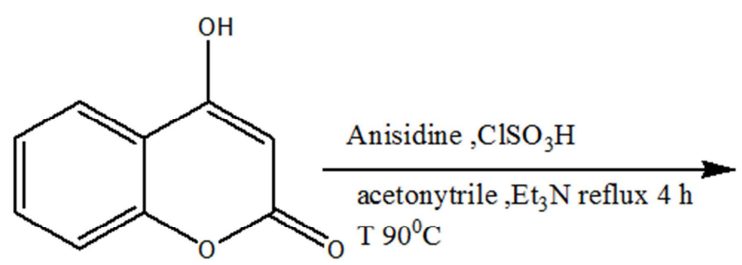

4-Hidroksil-kromen-2-one

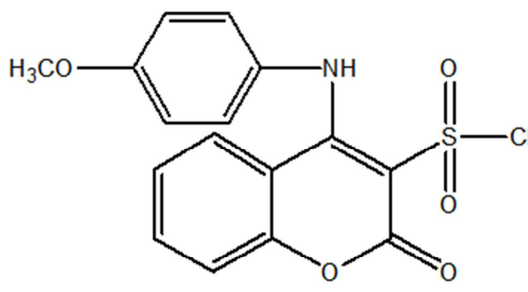

4-(4-Methoxy-phenylamino)-2-oxo-2Hchromene-3-sulfonyl chloride

Figure 1. Preparation of 4-(4-Methoxy-phenylamino)-2-oxo-2H-chromene-3-sulfonyl chloride (a).

Preparation ofN-\{3-[Diethoxy-(2-hydroxy-phenyl)methyl]-2-oxo-2H-chromen-4-yl $\}$-acetamide (b)

In a $100 \mathrm{ml}$ flask were mixed $3.5 \mathrm{~g}$ 4-hydroxy -Chomen2-one with $9 \mathrm{ml} \mathrm{C}_{2} \mathrm{H}_{5} \mathrm{OH}, 4 \mathrm{ml}$ Aldehyd salicylic, $3 \mathrm{~g}$
$\mathrm{CH}_{3} \mathrm{COONH}_{4}, 1 \mathrm{ml} \mathrm{HCl}$. The mixture was refluxed at $100^{\circ} \mathrm{C}$ for ca. $7 \mathrm{~h}$. The obtained yellow crystals are filtered and dried at room temperatur. Recrystallization form $\mathrm{C}_{2} \mathrm{H}_{5} \mathrm{OH}$ gave yellow crystals product of $78 \%$ yield, meltingpoint, $319^{\circ} \mathrm{C}$.<smiles>O=c1cc(O)c2ccccc2o1</smiles>

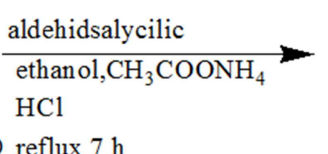<smiles>CCOC(OCC)(c1c(O)cccc1OC)c1c(NC(C)=O)c2ccccc2oc1=O</smiles>

4-Hydroxy-chromen-2-one $\mathrm{T} 100^{\circ} \mathrm{C}$

$N$ - $\{3$-[Diethoxy-(2-hydroxy-phenyl)-methyl]-2-oxo- $2 \mathrm{H}$ chromen-4-yl\}-acetamide

Figure 2. Preparation of $\mathrm{N}$-\{3-[Diethoxy-(2-hydroxy-phenyl)-methyl]-2-oxo-2H-chromen-4-yl\}-acetamide (b).

Preparation of 4-(6-Nitro-benzothiazol-2-ylamino)-2-oxo2H-chromene-3-carbaldehyde (c)

In a $100 \mathrm{ml}$ flask were mixed $2.5 \mathrm{~g}$ of 4-Hydroxy-chromen2-one, $2 \mathrm{~g}$ 2amino-6-nitro-benzotiazole, $8 \mathrm{ml} \mathrm{C}_{2} \mathrm{H}_{5} \mathrm{OH}$, with 4 $\mathrm{ml} \mathrm{HCOOH}$. The mixture was refluxed at $95^{\circ} \mathrm{C}$ in water bath for ca.3 h. The flask was placed in an ice bath for $1 \mathrm{~h}$ until yellow crystalline precipitate was formed. After filtration the product was recrystallized from $\mathrm{CH}_{3} \mathrm{CN}$. The recrystallizacion gave a yellow product at $90 \%$ yield, melting. point; $248^{\circ} \mathrm{C}$.<smiles>O=c1cc(O)c2ccccc2o1</smiles>

4-Hydroxy-chromen-2-one
2 amino-6-nitro-benzotiazol $8 \mathrm{ml}$ Ethanol, $\mathrm{HCOOH}$ $0.2 \mathrm{~m} 1 \mathrm{HCl}$ reflux $3 \mathrm{~h}$ $\mathrm{T} 95^{\circ} \mathrm{C}$

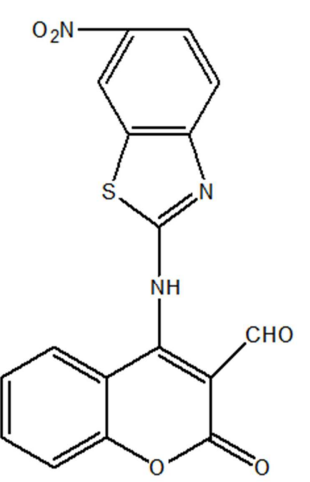

4-(6-Nitro-ben zothiazol-2-ylam ino)-2-oxo- $2 \mathrm{H}$ chromene-3-carbaldehyde

Figure 3. Preparation of 4-(6-Nitro-benzothiazol-2-ylamino)-2-oxo-2H-chromene-3-carbaldehyde (c). 
Table 1. Analytical data

\begin{tabular}{|c|c|c|c|c|c|c|c|c|}
\hline \multirow{2}{*}{ Compd } & \multirow{2}{*}{ Yeld \% } & \multirow{2}{*}{ m. $\mathbf{p}$} & \multirow{2}{*}{ M. F } & \multicolumn{5}{|c|}{ Elemental analysis. Calculatet: Found (calc) \% } \\
\hline & & & & $\mathbf{C}$ & $\mathbf{H}$ & $\mathbf{N}$ & $\mathbf{O}$ & $\mathbf{C l}$ \\
\hline \multirow[b]{2}{*}{ a } & \multirow{2}{*}{70} & \multirow{2}{*}{$310^{\circ} \mathrm{C}$} & \multirow{2}{*}{$\mathrm{C}_{16} \mathrm{H}_{12} \mathrm{ClNO}_{5} \mathrm{~S}$} & 52.54 & 3.31 & 3.33 & 21.87 & 9.69 \\
\hline & & & & 51.65 & 3.30 & 3.50 & 21.80 & 9.50 \\
\hline \multirow{2}{*}{$\mathrm{b}$} & \multirow{2}{*}{78} & \multirow{2}{*}{$319^{\circ} \mathrm{C}$} & \multirow{2}{*}{$\mathrm{C}_{22} \mathrm{H}_{23} \mathrm{NO}_{6}$} & 66.49 & 5.83 & 3.52 & 24.15 & \\
\hline & & & & 66.00 & 5,79 & 3.50 & 23.90 & \\
\hline \multirow{2}{*}{ c } & \multirow{2}{*}{90} & \multirow{2}{*}{$248^{\circ} \mathrm{C}$} & \multirow{2}{*}{$\mathrm{C}_{17} \mathrm{H}_{9} \mathrm{~N}_{3} \mathrm{O}_{5} \mathrm{~S}$} & 55.58 & 2.47 & 11.44 & 21.78 & \\
\hline & & & & 55.40 & 2.30 & 11.30 & 21.70 & \\
\hline
\end{tabular}

\subsection{Antibacterial Activity}

The purified synthesized compounds (a. b. c) was subjected to test in vitro its antibacterial activity against three bacterial cultures; Staphylococcus aureus, E coli and B. cereus. Antibacterial activity of compounds was investigated applying the Kirby-Bayer method or disc method $(\mathrm{d}=5.5 \mathrm{~mm}$ max. capacity $10 \mu \mathrm{g}$ )

Table 2. Antibacterial activity- Staphylococcus aureus.

\begin{tabular}{llll}
\hline \multirow{2}{*}{ Compound } & \multicolumn{3}{l}{ Inhibition zone $\mathbf{( m m})$} \\
\cline { 2 - 4 } & $\mathbf{2} \mathbf{~} \mathbf{g} / \mathbf{m l}$ & $\mathbf{3} \mathbf{~ m g} / \mathbf{m l}$ & $\mathbf{5} \mathbf{~ m g} / \mathbf{m l}$ \\
\hline $\mathrm{a}$ & 7 & 8,5 & 10,2 \\
$\mathrm{~b}$ & 7,7 & 8 & 11,3 \\
$\mathrm{c}$ & 8,2 & 9 & 12,7 \\
& & & \\
Cephalexine & 9 & 9 & $9-10 \mu \mathrm{g}$ \\
Streptomycine & 20 & 20 & $20-10 \mu \mathrm{g}$ \\
\hline
\end{tabular}

Table 3. Antibacterial activity - E. coli.

\begin{tabular}{llll}
\hline \multirow{2}{*}{ Compound } & \multicolumn{3}{l}{ Inhibition zone $(\mathbf{m m})$} \\
\cline { 2 - 4 } & $\mathbf{2} \mathbf{m g} / \mathbf{m l}$ & $\mathbf{3 m g} / \mathbf{m l}$ & $\mathbf{5 m g} \mathbf{m l}$ \\
\hline $\mathrm{a}$ & 10.4 & 13.7 & 18.8 \\
$\mathrm{~b}$ & 11 & 18.8 & 21.5 \\
$\mathrm{c}$ & 10 & 19 & 21.0 \\
& & & \\
Cephalexine & 9 & 9 & $9-10 \mu \mathrm{g}$ \\
Streptomycine & 23 & 23 & $23-10 \mu \mathrm{g}$ \\
\hline
\end{tabular}

Table 4. Antibacterial activity - Bacillus cereus.

\begin{tabular}{llll}
\hline \multirow{2}{*}{ Compound } & \multicolumn{3}{l}{ Inhibition zone (mm) } \\
\cline { 2 - 4 } & $\mathbf{2} \mathbf{m g} / \mathbf{m l}$ & $\mathbf{3 m g} \mathbf{m l}$ & $\mathbf{5 m g} \mathbf{m l}$ \\
\hline $\mathrm{a}$ & 9.2 & 14.4 & 20.8 \\
$\mathrm{~b}$ & 10.6 & 15.2 & 22.7 \\
$\mathrm{c}$ & 11 & 18.5 & 21.9 \\
& & & \\
Cephalexine & 9 & 9 & $9-10 \boldsymbol{\mu g}$ \\
Streptomycine & 23 & 23 & $23-10 \mu \mathrm{g}$ \\
\hline
\end{tabular}

\section{Results and Discussion}

By reacting equimolar amounts of 3-oxo-butiric acid ethyl ester and correspomding reagents (according scheme 1) under reflux reaction condictions product $1 \mathrm{a}$ is synthesized in $80 \%$ yield.

By reacting equimolar amounts of 4,7 Dyhidroxy chromen-2-one and corresponding reagents (according scheme 2) under reflux reaction condictions product $2 \mathrm{a}$ is synthesized in $70 \%$ yield.

By reacting equimolar amounts of 4,7 Dychloro-chromen2-one and corresponding reagents (according scheme 3) under reflux reaction condictions product $3 \mathrm{a}$ is synthesized in $70 \%$ yield.

The structure of 4,7 Dyhydroxy-chromen-2-one derivatives (a. b. c) were determined fromtheir IR, ${ }^{1} \mathrm{H}$ NMR, ${ }^{13} \mathrm{C}$ NMR spectar and their melting points as follows.

For (a); IR bands (KBr, cm-1) $3428 \mathrm{~cm}^{-1}$ (N-H stretch.), $3000 \mathrm{~cm}^{-1}$ (C-H aromatic), $2800 \mathrm{~cm}^{-1}$ (C-H stretch.), $1740 \mathrm{~cm}^{-}$ ${ }^{1}(\mathrm{C}=0$ stretch. $), 1600 \mathrm{~cm}^{-1}(\mathrm{~N}-\mathrm{H}) ; 1380 \mathrm{~cm}^{-1}\left(\mathrm{SO}_{2} \mathrm{Cl}\right) ; 720 \mathrm{~cm}^{-}$ ${ }^{1}$ (C-H aromatic)

${ }^{1} \mathrm{H}$ NMR (DMSO-d6) 8ppm;7.6-6.3 ( 6H aromatic);4.2 (H, $\mathrm{NH}) ; 3.73\left(\mathrm{H}, \mathrm{CH}_{3}\right)$

${ }^{13} \mathrm{C}$ NMR (DMSO) $\delta p p m ; 162(\mathrm{C}, \mathrm{C}=\mathrm{O}) ; 163.0$ ppm (C$\mathrm{NH}) ; \quad 150.8 \quad(\mathrm{C}-\mathrm{O}) ; \quad 152 \mathrm{ppm}(\mathrm{C}-\mathrm{O}) ; 114-128.1 \mathrm{ppm} \quad(6 \mathrm{C}$ aromatic);139.6ppm $(\mathrm{C}-\mathrm{N}) ; 56.0 \mathrm{ppm}\left(\mathrm{C}, \mathrm{CH}_{3}\right)$

For (b) IR bands (KBr, cm -1) $3400 \mathrm{~cm}^{-1}$ (OH stretch.); $3140 \mathrm{~cm}^{-1}$ (C-H aromatic.); $2936 \mathrm{~cm}^{1}$ ( C-H alifatic.); 1680 $\mathrm{cm}^{1} \quad$ (C-O stretch.); $1740 \mathrm{~cm}^{-1}$ (alfa pironi); $1380 \mathrm{~cm}^{-}$ ${ }^{1}(\mathrm{CONH}) ; 1220 \mathrm{~cm}^{-1}(\mathrm{C}-\mathrm{O}) ; 1150 \mathrm{~cm}^{-1}(\mathrm{CO}-\mathrm{N}) ; 748 \mathrm{~cm}^{-1}(\mathrm{C}-\mathrm{C}$ aromatic)

${ }^{1} \mathrm{H}$ NMR (DMSO-d6) $\delta p p m 8.0$ ppm(H, N-CO) 6.72-7.63 $(8 \mathrm{H}$ aromatic $) ; 5.0(\mathrm{H}, \mathrm{OH}) ; 3.41(4 \mathrm{H}, 2 \mathrm{CH}) ; 2.2\left(3 \mathrm{H}, \mathrm{CH}_{3}\right)$

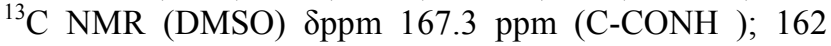
ppm $(\mathrm{C}, \quad \mathrm{C}=\mathrm{O}) ; 156.4 \mathrm{ppm} \quad(\mathrm{C}-\mathrm{OH}) ; 150.8$ ppm $(\mathrm{C}-\mathrm{O})$; 144.8(C-NH),54,6(C, $\left.\mathrm{CH}_{2}\right), 18.5$ ppm $\left(\mathrm{C}, \mathrm{CH}_{3}\right) ; 15.4(\mathrm{C}$, $\left.\mathrm{CH}_{3}\right) ; 121.3-129.0$ (8C aromatic)

For (c) IR bands (KBr, cm -1) $3380 \mathrm{~cm}^{-1}(\mathrm{~N}-\mathrm{H}$ stretch.);2990 $\mathrm{cm}^{-1}$ (C-H aromatic.); $2840 \mathrm{~cm}^{-1}$ (C-H aldehyde); $1740 \mathrm{~cm}^{-1}(\mathrm{C}=\mathrm{O}) ; 1620 \mathrm{~cm}^{-1}(\mathrm{~N}-\mathrm{H}), 1547 \mathrm{~cm}^{-1}(\mathrm{C}=\mathrm{N}) ; 1523(\mathrm{Ar}-$ $\left.\mathrm{NO}_{2}\right) ; 1256 \mathrm{ppm}(\mathrm{C}-\mathrm{O}$ stretch $) ; 720 \mathrm{ppm}(\mathrm{C}-\mathrm{H}$ aromatic $) ; 650$ ppm (C-S)

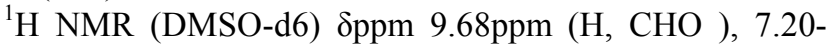
$9.05(7 \mathrm{H}$ aromatic $) ; 4.1(\mathrm{H}, \mathrm{NH})$

${ }^{13} \mathrm{CNMR}$ (DMSO) $\delta$ ppm 190.0ppm (C, CHO), 162ppm (C, $\mathrm{C}=\mathrm{O}) ; 181.1 \mathrm{ppm}(\mathrm{C}, \mathrm{C}-\mathrm{N}) ; 174.5 \mathrm{ppm}(\mathrm{C}-\mathrm{C}=\mathrm{N}) 150.8 \mathrm{ppm}$ (C, C-O); 121.3-126.6(10 C aromatic)

\section{Conclusion}

From the results the followin conclusion were drawn:The study provides the first evidence that compounds $(a, b, c)$ obviously inhibit the growth of S. auerus, E. coli and B. cereus.

The compounds $(\mathrm{a}, \mathrm{b}, \mathrm{c})$ compared with the antibacterial activity of Streptomycine in S. aureus, E. coli and B. cereus.

This study provided the first evidence that these 
compounds a, b, c showed a significant antibacterial effect against $\mathrm{S}$. aureus, E. coli and B. Cereus.

The chemical structures of synthesizen compounds were determined according to extensive NMR experiments and published data.

\section{Acknowledgements}

The authors thank Prof. Branko Stanovnik, University of Ljubljana and its laboratory staff for $1 \mathrm{H}$ NMR spectrum and elemental analyses.

\section{References}

[1] Govori. S, Kalaj. V, Rapic. V, Kalaj. L, and Dakovic. S, Heterocycel. Commun., 2002; 8,(129).

[2] Stanovnik. B, Susachitzky. H, and. Scriven. E. F, Progress in Heterocyclic Chemistry, Pergamon Press, Oxford, 1993; $5,(75-146)$.

[3] Lee. S. H, Shin, D. S, Kim. J. S, K.-B. Oh and Kan. S. S, Arch. Pharm. Res, 2003; (26).

[4] Vyas. K. B, NimavatK. S, Jani. G. R, and Hathi. M. V, Orbital, 2009; 1, (183).

[5] Abyshev. A. V, GimdeinV. A, Semenov E. V, E. MAgevE. M, Abdulla A. A, Zade and Gueseinov, Pharm. Chem. J., 2006; 40.(607).

[6] Aytemir. M. D, Hider. R. C, Erol. D. D, Ozalp. M and Ekizoglu. M, Turk. J. chem., 2003; 27: (445).
[7] El. Saghier. M. M, Naili. M. B, Rammash. M. Kh, Saleh. N. H and Kreddan. K. M, Arkivoc, 2007;(83).

[8] Nofa. Z. M, El-Zahar. M and Abd El Karim. M, Molecules, 2000; 5: (99).

[9] Chaluvaraju. K C and Ishwarbhat, K. Asian, J Chem 2008; 20, (4335).

[10] Ra Kali. R, S, Grworamma. J, and Suresh. B, Asian J Chem 2008: 20: (5289).

[11] Mohammed Ashraf. A and Mohammed. SH. Boorg Med Chem. Lett 2009; 17: (3314).

[12] Nofal. ZM, El-Zahar. M, Abd El-Karim. S, Novel coumarin derivatives with expected biological activity, Molecules. 2000; 5: (99- 113).

[13] Vyas. KB, Nimavat. KS, Jani. GR, Hathi. MV, Synthesis and microbial activity of coumarin derivatives metal complex: An in vitro evaluation. Orbital, 2009; 1: (183-192). Design, synthesis of novel azo derivatives of benzimidazole as potent antibacterial and anti tubercular agents

[14] A. A. Abou-Hussein, Wolfgang Linert, Synthesis, spectroscopic studies and inhibitory activity against bactria and fungi of acyclic and macrocyclic transition metal complexes containing a triamine coumarine Schiff base ligand Spectrochimica Acta Part A: Molecular and Biomolecular Spectroscopy, Volume 141, 15 April 2015, Pages 223-23.

[15] Danijela Lj Stojković, Verica V. Jevtić, Nenad Vuković, Milena Vukić, Srećko R. Trifunović, Synthesis, characterization, antimicrobial and antitumor reactivity of new palladium(II) complexes with methionine and tryptophane coumarine derivatives. Journal of Molecular Structure, Volume 1157, 5 April 2018, Pages 425-433. 